\title{
UTILIZAÇÃO DE REDES NEURAIS ARTIFICIAIS PARA A PROJEÇÃO DA DISTRIBUIÇÃO DIAMÉTRICA DE POVOAMENTO EQUIÂNEOS ${ }^{1}$
}

Mayra Luiza Marques da Silva Binoti², Daniel Henrique Breda Binoti³, Helio Garcia Leite ${ }^{4}$, Antonilmar Araújo Lopes da Silva ${ }^{5}$ e Claudio Pontes ${ }^{6}$

\begin{abstract}
RESUMO - Objetivou-se neste estudo desenvolver e avaliar a aplicação de redes neurais artificiais para a projeção de parâmetros da distribuição Weibull. Utilizaram-se dados de parcelas permanentes de eucaliptos, mensuradas em oito ocasiões. Ajustou-se a função Weibull com dois parâmetros para todas as parcelas e ocasiões, pelo método da máxima verossimilhança. A projeção da distribuição diamétrica foi feita através de redes neurais artificiais. Comparou-se o método proposto com o método tradicionalmente utilizado na modelagem da distribuição diamétrica. Os modelos utilizando RNA apresentaram melhorias na dispersão gráfica dos resíduos, bem como das estatísticas avaliadas. O método proposto mostrou-se superior ao método comumente usado.
\end{abstract}

Palavras-chave: Weibull; Eucalyptus; Inteligência artificial.

\section{USE OF ARTIFICIAL NEURAL NETWORK FOR DIAMETER DISTRIBUTION MODELLING FOR EVEN-AGED POPULATION}

\begin{abstract}
The aim of this study is to develop and evaluate the application of artificial neural networks to forecast parameters of the Weibull distribution. We used data from permanent plots of eucalyptus, measured on eight occasions. We adjusted the Weibull function with two parameters for all plots and occasions, by the method of maximum likelihood. The projection of the diameter distribution was made through artificial neural networks. We compared the proposed method to the method traditionally used in modeling the diameter distribution. The models using ANN presented improvements in the graphic dispersion of the residues, as well as the evaluated statistics. The proposed method was superior to the commonly used method.
\end{abstract}

Keywords: Weibull; Eucalyptus; Artificial intelligence.

\footnotetext{
${ }^{1}$ Recebido em 11.11.2010 aceito para publicação em 05.06.2014

${ }^{2}$ Departamento de Engenharia Florestal, Universidade Federal dos Vales do Jequitinhonha e Mucuri, UFVJM, Brasil. E-mail: $<$ mayrabinoti@gmail.com>.

${ }^{3}$ Programa de Pós-Graduação, Universidade Federal de Viçosa, UFV, Brasil. E-mail: <danielhbbinoti@gmail.com>.

${ }^{4}$ Departamento de Engenharia Florestal, Universidade Federal de Viçosa, UFV, Brasil. E-mail: <hgleite@gmail.com>.

${ }^{5}$ Celulose Nipo Brasileira - CENIBRA. E-mail: <antonilmar.silva@cenibra.com.br>.

${ }^{6}$ UNESCO/MCTI/CEMADEN.
} 


\section{INTRODUÇÃO}

O conhecimento da estrutura diamétrica de um povoamento florestal é de grande importância para os objetivos do manejo de florestas equiâneas. Essa estrutura permite inferir sobre o volume total do povoamento, produção, sortimentos de madeira e fase de desenvolvimento do povoamento, sendo seu conhecimento fundamental para a análise econômica de povoamentos submetidos a desbastes, bem como a simulação de regimes de manejo (CLUTTER et al., 1983; CAMPOS; LEITE, 2009).

Diferentes distribuições teóricas, como beta, Weibull, Johnson SB e a função Hyper têm sido utilizadas para descrever a estrutura diamétrica de povoamentos florestais (BAILEY; DELL, 1973; LOETSCH et al., 1973; HAFLEY; SCHREUDER, 1977; LEITE et al., 2010). A distribuição pode ser ajustada em termos de frequência de árvores ou área basal por hectare (GOVE; PATIL, 1998; SIIPILEHTO, 1999). Desses, a distribuição de diâmetro com base nas frequências de árvore é a abordagem mais amplamente utilizada no Brasil.

Para projeção da distribuição, dois métodos principais podem ser aplicados, ou seja, o método de predição ou projeção dos parâmetros e o método de recuperação de parâmetros (HYINK, 1980; HYINK; MOSER, 1983; GUIMARÃES, 1994). Na predição de parâmetros, os parâmetros de uma função densidade de probabilidade, a exemplo da distribuição Weibull, são obtidos por modelos de regressão em função de características do povoamento (CAMPOS; LEITE, 2009). No método de recuperação de parâmetros, os parâmetros da função de distribuição são obtidos em função de um sistema de equações fundamentado em medidas de posição. Entre as formas de recuperação de estimativas da distribuição, destacam-se o método dos percentis da distribuição de diâmetro (BAILEY et al., 1981; CAO; BURKHART, 1984; GUIMARÃES, 1994; CAO, 2004) ou momentos da distribuição de diâmetro (NEWBY, 1980; BURK; NEWBERRY, 1984; CAO, 2004).

A computação neurobiológica tem sido empregada em alguns estudos na Ciência Florestal (DIAMONTOPOULOU, 2005; GÖRGENS, 2006; SILVA, 2008). Redes neurais artificiais (RNA) são processadores paralelamente distribuídos compostos por unidades de processamento simples (neurônios artificiais), que armazenam conhecimento experimental (aprendizagem), tornando-o disponível para uso (generalização) (BRAGA et al., 2000; HAYKIN, 2001; BINOTI, 2010). Autilização de redes neurais se aplica, principalmente, aos problemas de aproximação de função, classificação de padrões, agrupamento de dados, predição (séries temporais), otimização, recuperação de conteúdo e controle (JAIN et al., 1996; HAYKIN, 2001; BINOTI, 2010).

A projeção de parâmetros de uma função de densidade probabilidade que descreva a estrutura diamétrica de um povoamento florestal se enquadra na tarefa de aproximação de função que consiste em projetar uma rede neural que aproxime a função desconhecida $f(x)$, que descreve o mapeamento dos pares de entrada-saída $\left\{\left(x_{1}, y_{1}\right),\left(x_{2}, y_{2}\right), \ldots,\left(x_{n}, y_{n}\right)\right\}$ de um conjunto de $n$ padrões de treinamento.

Objetivou-se neste estudo desenvolver e avaliar a aplicação de redes neurais artificiais para a projeção de parâmetros da distribuição Weibull, bem como comparar o método proposto com modelos de distribuição diamétrica comumente utilizados na área florestal.

\section{MATERIAL E MÉTODOS}

\subsection{Dados}

Os dados utilizados neste estudo foram obtidos de aproximadamente 10.000 parcelas retangulares permanentes de $340 \mathrm{~m}^{2}$, instaladas em povoamentos de híbridos de Eucalyptus grandis x Eucalyptus urophylla, na região Centro-Oeste do Estado de Minas Gerais. Esses povoamentos foram estabelecidos sob arranjo espacial de $3 \times 3 \mathrm{~m}$, sendo a madeira utilizada para produção de polpa de celulose de fibra curta. Na idade de corte, aproximadamente aos 7 anos, a produtividade média nesses povoamentos varia de 25 nos piores locais a $50 \mathrm{~m}^{3} \cdot \mathrm{ha}^{-1}$. $\mathrm{ano}^{-1}$ nos melhores. As medições de diâmetros foram efetuadas nas árvores com dap acima de $5 \mathrm{~cm}$, nas idades médias de 28, 40, 52, 64, 76, 88, 100 e 112 meses. O banco de dados foi separado em dois conjuntos de dados, sendo um para construção do modelo e outro para sua validação. Nessa separação, foi mantida uma frequência homogênea de dados por classe de capacidade produtiva.

\subsection{Estrutura diamétrica}

Para a construção dos modelos de distribuição diamétrica, ajustou-se a função Weibull para todas as parcelas e ocasiões. A função Weibull pode ser definida como: 


$$
f(X)=\left\{\frac{\gamma}{\beta}\left(\frac{(x)}{\beta}\right)^{(\gamma-1)} e^{-\left(\frac{(X)}{\beta}\right)^{\gamma}}\right.
$$

em que $\beta$ é o parâmetro de escala $(\beta>0)$, $\gamma$ é o parâmetro de forma ( $(\tilde{a}>0)$ e x é o centro de classe de diâmetro $(x>0)$. Para realizar os ajustes, os dados de distribuição diamétrica observados em cada parcela, em todas as medições, foram agrupados em classes com amplitude do 1,0 cm. Procedeu-se ao ajuste das funções pelo Método da Máxima Verossimilhança (BAILEY; DELL, 1973).

Testou-se a aderência das funções aos dados pelo teste de Kolmogorov-Smirnorv (SOKAL; ROHLF, 1981; GIBBONS; SUBHABRATA, 1992). Esse teste compara a frequência acumulada estimada com a observada, sendo a classe de maior divergência a estatística do teste $(d n)$. O teste foi aplicado a cada ajuste com nível de significância 1\%. Nesse teste, quanto maior o valor de $d n$, maior a divergência entre os valores reais e os valores estimados pela função.

\subsection{Treinamento e generalização das redes neurais artificiais}

O treinamento de uma RNA é o processo de ajuste de seus pesos através de um algoritmo de aprendizagem, que extrai características dos dados fornecidos e tem por objetivo gerar uma rede que desempenhe a tarefa de interesse (BINOTI, 2010).

Foram treinadas redes para projetar o parâmetro de forma, parâmetro de escala, diâmetro mínimo e diâmetro máximo. Os parâmetros e características do povoamento foram projetados em função das seguintes características: idade atual e futura, altura e diâmetro mínimo, máximo, médio, diâmetro quadrático e altura dominante, bem como os parâmetros da função na idade atual. Nessa etapa, foram utilizados dados de aproximadamente 5.000 parcelas de inventário.

Foram treinadas 100 redes na estrutura de Perceptrons de Múltiplas Camadas (MLP) para projeção dos parâmetros de interesse. Nessa etapa do estudo, utilizou-se a ferramenta Intelligent Problem Solver (IPS) do software Statistica 7 (STATSOFT, 2010). Diante do grande número de possíveis combinações das variáveis de entrada disponíveis, foi solicitada a otimização desta seleção por algoritmos do IPS. Contudo, para fins de operacionalização da técnica proposta, selecionaram-se somente quatro redes, sendo uma com as variáveis mínimas necessárias (parâmetro na idade atual e idades atual e futura), duas com as variáveis selecionadas pelo otimizador e uma com a inclusão de todas as variáveis disponíveis.

A generalização é a capacidade de uma rede neural produzir saídas adequadas para entradas que não estavam presentes durante o treinamento (aprendizagem). Nesta etapa, foram utilizados dados de aproximadamente 5.000 parcelas de inventário não utilizados durante o treinamento.

\subsection{Modelo de distribuição de diâmetros}

O modelo de distribuição diamétrica utilizado neste trabalho foi proposto por Nogueira et al. (2005) e é composto pelas seguintes equações:

$$
\begin{aligned}
& \beta_{2}=\beta_{1}\left(\frac{I_{1}}{I_{2}}\right)+\alpha_{0}\left(1-\frac{I_{1}}{I_{2}}\right) d \max _{2} \\
& \operatorname{Ln} \gamma_{2}=\operatorname{Ln} \gamma_{1} e^{\left(-\left(\alpha_{0}\right)\left(I_{2}^{\alpha_{1}}-I_{1}^{\alpha_{1}}\right)\right)} \\
& d \max _{2}=d \max _{1}\left(\frac{I_{1}}{I_{2}}\right)+\alpha_{0}\left(1-\frac{I_{1}}{I_{2}}\right) \beta_{1} \\
& d \min _{2}=\operatorname{dmin}_{1} \mathrm{e}^{\left(-\left(-\alpha_{0}\right)\left(I_{2}^{\alpha_{1}}-I_{1}^{\alpha_{1}}\right)\right)}
\end{aligned}
$$

em que $I_{1}$ e $I_{2}$ são as idades atual e futura, respectivamente, em meses; $\gamma_{1}$ e $\gamma_{2}$ são os parâmetros de forma da função Weibull nas idades atual e futura; $\beta_{1}$ e $\beta_{2}$ são os parâmetros de escala da função Weibull nas idades atual e futura; $d \max _{1}$ e $d m a x_{2}$ são os diâmetros máximos nas idades atual e futura, em cm; $d \min _{1}$ e dmin $_{2}$ são os diâmetros mínimos nas idades atual e futura, em cm; e Ln indica o logaritmo neperiano.

Após os ajustes de ambas as funções para cada parcela em cada período, procedeu-se ao ajuste das funções que compõem o modelo de distribuição diamétrica (MDD) pelo método iterativo Quasi-Newton, empregando o software Statistica 9 (STATSOFT, 2010). A distribuição diamétrica foi projetada a partir de uma distribuição em uma idade atual para uma idade futura; a primeira 
projeção foi feita a partir da média das frequências observadas em cada classe da distribuição diamétrica. As projeções subsequentes foram feitas sobre as projeções imediatamente anteriores. Em razão da baixa mortalidade apresentada nos dados do estudo, não se realizou a projeção do número de árvores, sendo este considerado constante.

\subsection{Avaliação das projeções das distribuições diamétricas}

A redistribuição teórica dos diâmetros foi avaliada pela capacidade das redes neurais e do sistema de equações em projetar os diâmetros máximo e mínimo e os parâmetros da função Weibull e hiperbólica ( $\hat{a}$ e ã) em qualquer idade. O coeficiente de correlação entre os valores observados e estimados de frequência por classe de diâmetro e a análise gráfica dos resíduos foram utilizados para avaliar a exatidão das estimativas de frequência por classe de diâmetro. Avaliaram-se a consistência, a capacidade de projeção e a realidade biológica do sistema e das RNA, projetando diferentes distribuições iniciais observadas, para idades futuras, bem como se procedeu à comparação com os respectivos valores observados de frequência por classe de diâmetro.

A avaliação das estimativas geradas pelas RNA nas três etapas - treinamento, generalização e prognose - e do modelo de distribuição diamétrica foi feita mediante o emprego de estatísticas e da análise gráfica de resíduos. Essa análise gráfica consistiu na inspeção estatística da dispersão dos erros (resíduos) percentuais em relação aos valores observados, sendo:

$$
\text { erro }_{\%}=\frac{(\hat{Y}-Y)}{Y} .100
$$

em que $Y$ é a saída observada nos inventários e $\hat{Y}$ é a saída estimada.

As estatísticas empregadas foram a correlação entre os valores estimados e observados dos parâmetros projetados e a raiz do erro quadrado médio (RMSE\%).

$$
r_{Y \hat{Y}}=\frac{\operatorname{cov}(Y, \hat{Y})}{\sqrt{s^{2}(Y) s^{2}(\hat{Y})}}
$$

$e$ m que $s^{2}$ é a variância e cov, a covariância.

$$
\operatorname{RMSE}(\%)=\frac{100}{\bar{Y}} \sqrt{\frac{\sum_{i=1}^{n}\left(Y_{i}-\hat{Y}_{i}\right)^{2}}{n}}
$$

em que $\bar{Y}$ é a média da saída observada e $n$, o número total de dados.

\section{RESULTADOS}

Ajustou-se a função Weibull para todas as parcelas e em cada situação, sendo cada estimação comparada com a distribuição observada. Todos os ajustes resultaram em aderência aos dados pelo teste de KolmogorovSmirnorv $(\mathrm{P}>0,01)$.

As características das 16 redes treinadas, bem como as estatísticas utilizadas na sua avaliação, são apresentadas na Tabela 1. Entre as redes treinadas, escolheram-se uma rede para a projeção de cada variável de interesse e a análise gráfica de resíduos. Os gráficos de dispersão dos resíduos e histogramas de erros (\%) são apresentados na Figura 1.

O teste $\mathrm{t}$ foi significativo para todos os parâmetros em todas as equações das equações que compõem o modelo de distribuição diamétrica. Todas as equações apresentaram valores reativamente altos de coeficientes de correlação entre valores observados e estimados, indicando que as variáveis independentes utilizadas nas equações têm grande influência nas variáveis dependentes. A análise gráfica dos resíduos permitiu inferir a inexistência de tendenciosidade nos ajustes (Figura 1). Os modelos ajustados e os respectivos coeficientes de correlação e RMSE (\%) são apresentados a seguir:

$$
\begin{aligned}
& \beta_{2}=\beta_{1}\left(\frac{I_{1}}{I_{2}}\right)+\alpha_{0}\left(1-\frac{I_{1}}{I_{2}}\right) d \max _{2} \\
& R_{\hat{y} y}=0,964 \quad \operatorname{RMSE}(\%)=15,0200 \\
& \operatorname{Ln} \gamma_{2}=\operatorname{Ln} \gamma_{1} e^{\left(-(0,1687)\left(I_{2}^{0,2564}-I_{1}^{0,2564}\right)\right)} \\
& R_{\hat{y} y}=0,8982 \quad \operatorname{RMSE}(\%)=29,207
\end{aligned}
$$

$$
\begin{aligned}
& d \max _{2}=d \max _{1}\left(\frac{I_{1}}{I_{2}}\right)+\alpha_{0}\left(1-\frac{I_{1}}{I_{2}}\right) \beta_{1} \\
& R_{\hat{y} y}=0,9438 \quad \operatorname{RMSE}(\%)=4,9460
\end{aligned}
$$

$$
\begin{aligned}
& d \min _{2}=d \min _{1} \mathrm{e}^{\left(-\left(-\alpha_{0}\right)\left(I_{2}^{\alpha_{1}}-I_{1}^{\alpha_{1}}\right)\right)} \\
& R_{\hat{y} y}=0,8841 \quad \operatorname{RMSE}(\%)=8,7811
\end{aligned}
$$


Tabela 1 - Características e estatísticas das redes neurais artificiais treinadas para projetar os parâmetros da função densidade de probabilidade Weibull e as variáveis do povoamento.

Table 1 - Characteristics and statistics of artificial neural networks trained to design the parameters of the Weibull probability density function and population variables.

\begin{tabular}{|c|c|c|c|c|c|c|c|c|}
\hline$\overline{R N A}$ & Arquitetura* & $\mathrm{r}$ & $\begin{array}{l}\text { Índice do } \\
\text { treino }^{* *}\end{array}$ & $\begin{array}{l}\text { Índice da } \\
\text { seleção** }^{* *}\end{array}$ & $\begin{array}{l}\text { Índice da } \\
\text { validação }^{* *}\end{array}$ & $\begin{array}{l}\text { Variáveis de entrada } \\
\text { de saída }\end{array}$ & Variável & RMSE\% \\
\hline 1 & $3-9-1$ & 0,9650 & 0,27 & 0,26 & 0,26 & $\beta_{1}, I_{1}, I_{2}$ & $\beta_{2}$ & 3,30 \\
\hline 2 & $9-5-1$ & 0,9724 & 0,23 & 0,23 & 0,24 & $\begin{array}{c}\beta_{1}, \gamma_{1}, I_{1}, \operatorname{Hmin}_{1}, \operatorname{Hd}_{1}, \operatorname{Hmed}_{1}, \\
\operatorname{Hmax}_{1}, \operatorname{dapmax}_{1}, \mathrm{~N}_{1}, I_{2}\end{array}$ & $\beta_{2}$ & 1,71 \\
\hline 3 & $11-5-1$ & 0,9720 & 0,23 & 0,23 & 0,25 & $\begin{array}{c}\beta_{1}, \gamma_{1}, \mathrm{q}_{1}, \mathrm{I}_{1}, \operatorname{Hmin}_{1}, \operatorname{Hmed}_{1}, \\
\operatorname{Hmax}_{1}, \text { dapmin }_{1}, \text { dapmax }_{1}, \mathrm{~N}_{1}, \mathrm{I}_{2}\end{array}$ & $\beta_{2}$ & 0,97 \\
\hline 4 & $13-13-1$ & 0,9728 & 0,23 & 0,24 & 0,23 & $\begin{array}{c}\beta_{1}, \gamma_{1}, \mathrm{q}_{1}, \mathrm{I}_{1}, \operatorname{Hmin}_{1}, \mathrm{Hd}_{1}, \mathrm{Hmed}_{1}, \\
\operatorname{Hmax}_{1}, \text { dapmin }_{1}, \text { dapmed }_{1}, \\
\operatorname{dapmax}_{1}, \mathrm{~N}_{1}, \mathrm{I}_{2}\end{array}$ & $\beta_{2}$ & 1,21 \\
\hline 5 & $3-9-1$ & 0,9541 & 0,29 & 0,31 & 0,30 & $\operatorname{Ln}\left(\gamma_{1}\right), I_{1}, I_{2}$ & $\operatorname{Ln}\left(\gamma_{2}\right)$ & 7,24 \\
\hline 6 & $9-7-1$ & 0,9549 & 0,30 & 0,30 & 0,30 & $\begin{array}{l}\operatorname{Ln}\left(\gamma_{1}\right), \mathrm{q}_{1}, \mathrm{I}_{1}, \mathrm{Hd}_{1}, \operatorname{Hmed}_{1}, \\
\operatorname{Hmax}_{1}, \text { dapmax }_{1}, \mathrm{~N}_{1}, \mathrm{I}_{2}\end{array}$ & $\operatorname{Ln}\left(\gamma_{2}\right)$ & 7,17 \\
\hline 7 & $10-6-1$ & 0,9566 & 0,29 & 0,29 & 0,29 & $\begin{array}{l}\operatorname{Ln}\left(\gamma_{1}\right), \mathrm{q}_{1}, \operatorname{Hmin}_{1}, \operatorname{Hd}_{1}, \operatorname{Hmed}_{1} \\
\operatorname{Hmax}_{1}, \operatorname{dapmin}_{1}, \operatorname{dapmax}_{1}, \mathrm{~N}_{1}, \mathrm{I}_{2}\end{array}$ & $\operatorname{Ln}\left(\gamma_{2}\right)$ & 7,04 \\
\hline 8 & $13-13-1$ & 0,9581 & 0,28 & 0,29 & 0,29 & $\begin{array}{c}\beta_{1}, \operatorname{Ln}\left(\gamma_{1}\right), \mathrm{q}_{1}, \mathrm{I}_{1}, \operatorname{Hmin}_{1}, \mathrm{Hd}_{1}, \\
\operatorname{Hmed}_{1}, \operatorname{Hmax}_{1}, \operatorname{dapmin}_{1}, \\
\operatorname{dapmed}_{1}, \operatorname{dapmax}_{1}, \mathrm{~N}_{1}, \mathrm{I}_{2}\end{array}$ & $\operatorname{Ln}\left(\gamma_{2}\right)$ & 6,92 \\
\hline 9 & $3-6-1$ & 0,9414 & 0,33 & 0,32 & 0,33 & $\mathrm{I}_{1}$, dapmin $_{1}, \mathrm{I}_{2}$ & dapmin $_{2}$ & 8,71 \\
\hline 10 & $7-6-1$ & 0,9492 & 0,31 & 0,32 & 0,33 & $\begin{array}{l}\mathrm{q}_{1}, \mathrm{I}_{1}, \mathrm{Hd}_{1}, \text { dapmin }_{1}, \\
\text { dapmed }_{1}, \mathrm{~N}_{1}, \mathrm{I}_{2}\end{array}$ & dapmin $_{2}$ & 8,13 \\
\hline 11 & $8-3-1$ & 0,9498 & 0,30 & 0,32 & 0,32 & $\begin{array}{l}\mathrm{I}_{1}, \mathrm{Hd}_{1}, \operatorname{Hmed}_{1}, \operatorname{Hmax}_{1} \\
\operatorname{dapmin}_{1}, \operatorname{dapmax}_{1}, \mathrm{~N}_{1}, \mathrm{I}_{2}\end{array}$ & dapmin $_{2}$ & 8,08 \\
\hline 12 & $11-12-1$ & 0,9512 & 0,31 & 0,32 & 0,29 & $\begin{array}{c}\mathrm{q}_{1}, \mathrm{I}_{1}, \operatorname{Hmin}_{1}, \mathrm{Hd}_{1}, \operatorname{Hmed}_{1} \text {, } \\
\operatorname{Hmax}_{1}, \operatorname{dapmin}_{1}, \text { dapmed }_{1} \text {, } \\
\operatorname{dapmax}_{1}, \mathrm{~N}_{1}, \mathrm{I}_{2}\end{array}$ & dapmin $_{2}$ & 7,97 \\
\hline 13 & $3-9-1$ & 0,9772 & 0,21 & 0,21 & 0,22 & $\mathrm{I}_{1}$, dapmax ${ }_{1}, \mathrm{I}_{2}$ & dapmax $_{2}$ & 3,46 \\
\hline 14 & $6-7-1$ & 0,9804 & 0,20 & 0,19 & 0,20 & $\mathrm{q}_{1}, \mathrm{I}_{1}, \mathrm{Hd}_{1}, \mathrm{Hmax}_{1}$, dapmax ${ }_{1}, \mathrm{I}_{2}$ & dapmax $_{2}$ & 3,20 \\
\hline 15 & $7-8-1$ & 0,9806 & 0,20 & 0,19 & 0,20 & $\begin{array}{c}\mathrm{I}_{1}, \operatorname{Hmed}_{1}, \operatorname{Hmax}_{1}, \text { dapmin }_{1} \text {, } \\
\text { dapmax }_{1}, \mathrm{~N}_{1}, \mathrm{I}_{2}\end{array}$ & dapmax $_{2}$ & 3,19 \\
\hline 16 & $11-12-1$ & 0,9811 & 0,19 & 0,20 & 0,20 & $\begin{array}{l}\mathrm{q}_{1}, \mathrm{I}_{1}, \operatorname{Hmin}_{1}, \mathrm{Hd}_{1}, \operatorname{Hmed}_{1}, \operatorname{Hmax}_{1} \text {, } \\
\text { dapmin }_{1}, \operatorname{dapmed}_{1}, \operatorname{dapmax}_{1}, \mathrm{~N}_{1}, \mathrm{I}_{2}\end{array}$ & $\operatorname{dapmax}_{2}$ & 3,15 \\
\hline
\end{tabular}

* Número de neurônios em cada camada. ** Índices do treino (obtenção da rede), seleção (parada do treino) e validação (qualidade da rede treinada) que devem ser estáveis, ou seja, pouca variação entre eles. $\beta_{1}=$ parâmetro $\beta$ da função Weibull em $\mathrm{I}_{1}, \gamma_{1}=$ parâmetro $\gamma$ da função

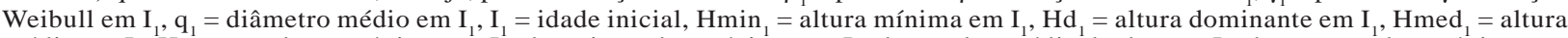

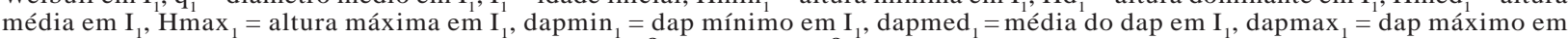
$\mathrm{I}_{1}, \mathrm{~N}_{1}=$ número de árvores/ha em $\mathrm{I}_{1}, \mathrm{I}_{2}=$ idade futura, $\beta_{2}=$ parâmetro $\beta$ da função Weibull em $\mathrm{I}_{2}, \gamma_{2}=$ parâmetro $\gamma$ da função Weibull em $\mathrm{I}_{2} \cdot \mathrm{r}=$ correlação entre os valores da variável estimada e observada.

\section{DISCUSSÃO}

Apesar da existência de diversas funções estatísticas, de diferentes métodos para ajuste dessas funções e de alternativas para estimação de distribuições diamétricas futuras, o enfoque de modelagem tem sido o mesmo, com predomínio de uma das formas da função Weibull (HYINK, 1980; CAO, 2004; LEITE et al., 2010). Entre outras características, a boa correlação apresentada por seus parâmetros com características do povoamento tem sido fundamental para a utilização dessa função (GUIMARÃES, 1994).
O processo de modelagem das distribuições diamétricas envolve basicamente a análise das alterações nos coeficientes, ou medidas de posição da função de distribuição, as quais são determinadas pelo crescimento do povoamento florestal. De modo geral, os modelos usuais de distribuição de diâmetros têm apresentado resultados satisfatórios, apenas no que concerne à estimativa do parâmetro de escala. Estudos na área de modelagem da distribuição diamétrica têm demonstrado que a projeção do parâmetro de forma apresenta baixa correlação com características do povoamento (GUIMARÃES, 1994). 


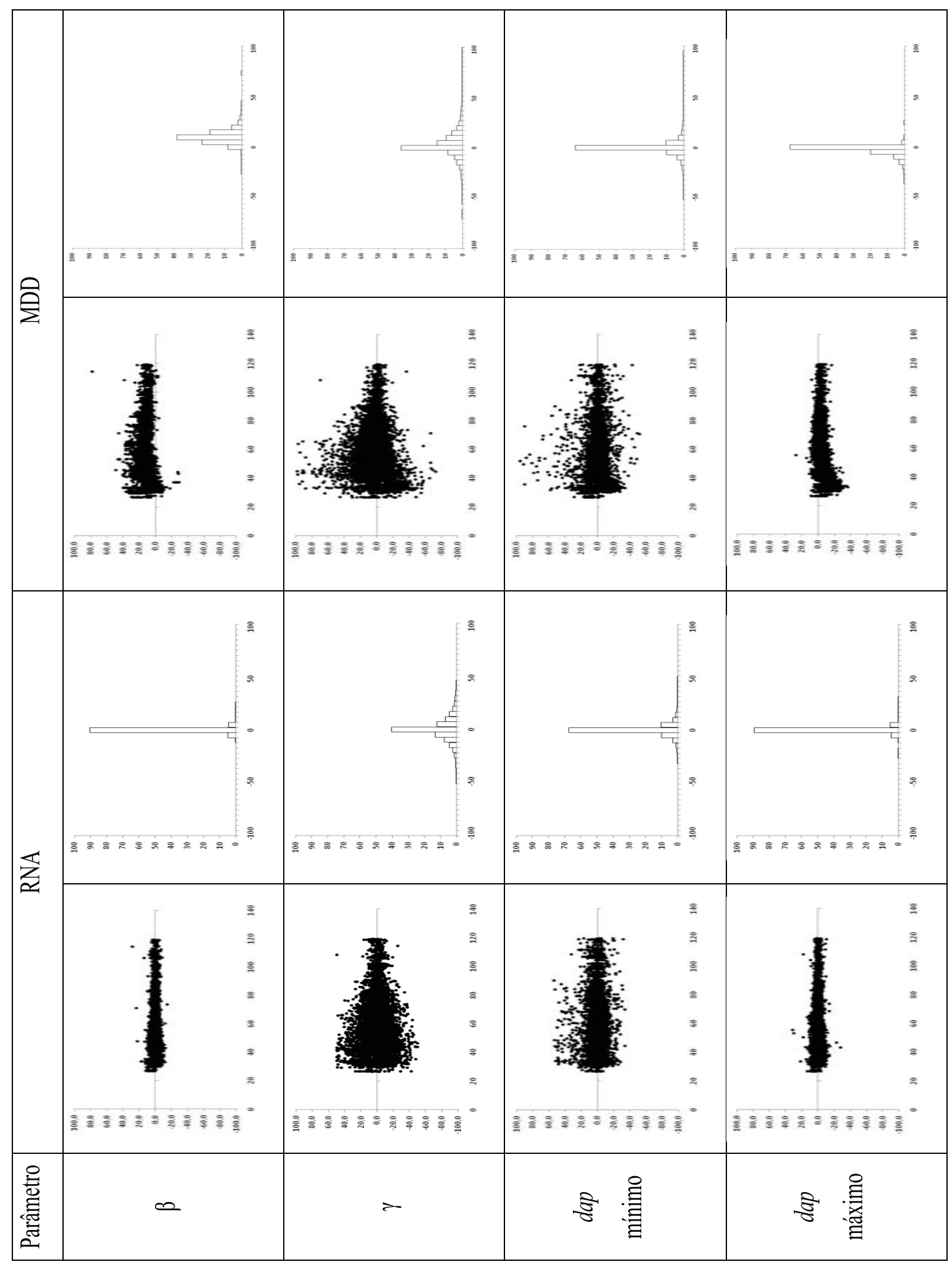

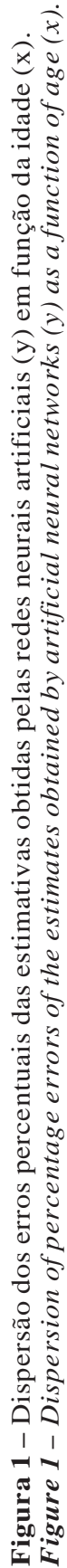

Revista Árvore, Viçosa-MG, v.38, n.4, p.747-754, 2014 
Neste estudo, foi proposto um método alternativo para modelagem de distribuições diamétricas que não envolve o uso de modelos de regressão para a projeção da distribuição diamétrica em idades futuras. A proposta consiste na projeção dos parâmetros da distribuição diamétrica para idades futuras em função de características do povoamento, através de técnicas de redes neurais artificiais. A principal vantagem do método proposto se baseia na eficiência da modelagem de relações não lineares que pode haver entre as características do povoamento e os parâmetros da $f d p$. Outra vantagem se refere à inclusão de variáveis categóricas nos modelos, como tipo de solo, relevo, projeto etc., que, apesar de não utilizados neste estudo, são apresentadas por Binoti (2010).

Foi comprovada a maior eficiência da modelagem via RNA sobre as equações que compõem o modelo de distribuição diamétrica na forma mais usual (Figura 1). Conforme resultado deste estudo, o enfoque de modelagem resulta em estimativas livres de bias.

As variáveis de entrada para as redes neurais são de fácil obtenção em rotinas de inventário florestal contínuo (Tabela 1), viabilizando o emprego da metodologia proposta neste estudo, em que foi utilizado o software Statistica (STATSOFT, 2010) para treinamento das redes. Porém, o algoritmo de treinamento utilizado está disponível em outros softwares, incluindo o software livre NeuroForest (BINOTI et al., 2012). Isso viabiliza o emprego da metodologia por diferentes usuários. A existência de diferentes algoritmos de treinamento no Neuroforest pode resultar em maior eficiência na modelagem do que aquela encontrada neste estudo. Da mesma forma, a inclusão de variáveis de entrada categóricas pode aumentar a exatidão das estimativas. Esse ganho de exatidão já foi verificado em modelagem em nível de povoamento por Binoti (2010).

A metodologia proposta neste estudo, de emprego de redes neurais para estimação dos parâmetros da distribuição diamétrica em idade futura, pode ser empregada para outras funções de densidade probabilidade, como as funções descritas por Binoti (2008). Assim, para outros estudos, sugere-se a incorporação de características edafoclimáticas que, apesar das relações aparentemente desconhecidas em relação à variação dos parâmetros da função Weibull, podem ser modeladas pelas RNA.

\section{CONCLUSÃO}

A modelagem de distribuições de diâmetros em povoamentos equiâneos por meio de redes neurais é mais eficiente do que a modelagem usual, feita pelo emprego de modelos de regressão.

\section{REFERÊNCIAS}

BAILEY, R. L.; ABERNATHY, N. C.; JONES, E. P. Diameter distributions models for repeatedly thinned slash pine plantations. Washington: USDA Forest Service Gen. Tech. Rep. SO-34, 1981.

BAILEY, R. L.; DELL, T. R. Quantifying diameter distributions with the Weibull function. Forest Science, v.19, n.2, p.97-104, 1973.

BINOTI, M. L. M. S. Redes neurais artificiais para prognose da produção de povoamentos não desbastados de eucalipto. 2010. 54f. Dissertação (Mestrado em Ciência Florestal) - Universidade Federal de Viçosa, Viçosa, MG, 2010.

BRAGA, A. P.; LUDERMIR, T. B.; CARVALHO, A P. L. F. Redes neurais artificiais: teoria e aplicações. Rio de Janeiro: LTC, 2000. 262 p.

BURK, T.; NEWBERRY, J. A simple algorithm for moment-based recovery of Weibull distribution parameters. Forest Science, v.30, n.2, p.329-332, 1984.

CAMPOS, J. C. C.; LEITE, H. G. Mensuração florestal: perguntas e respostas. 3.ed. Viçosa, MG: Universidade Federal de Viçosa, 2009. 548p.

CAO, Q. V. Predicting parameters of a Weibull function for modeling diameter distribution. Forest Science, v.50, n.4, p.682-685, 2004.

CAO, Q.; BURKHART, H. A segmented distribution approach for modeling diameter frequency data. Forest Science, v.30, n.1. p.129-137, 1984.

CLUTTER, J. L. et al. Timber management: a quantitative approach. New York: John Willey e Sons, 1983. 333p.

Revista Árvore, Viçosa-MG, v.38, n.4, p.747-754, 2014 
DIAMANTOPOULOU, M. J. Artificial neural networks as an alternative tool in pine bark volume estimation. Computers and Electronics in Agriculture, v.10, p.235-244, 2005.

GIBBONS, J. D.; SUBHABRATA, C. Nonparametric statistical inference. 3.ed. New York: Marcel Dekker, 1992. 544p. (Statistics: Textbook and Monograph, v.31).

GÖRGENS, E. Estimação do volume de árvores utilizando redes neurais artificiais. 2006. 84f. (Mestrado em Ciência Florestal) - Universidade Federal de Viçosa, Viçosa, MG, 2006.

GOVE, J. H.; PATIL, G. P. Modelling basal area size distribution of forest stands: a compatible approach. Forest Science, v. 44, n.2, p.285-297, 1998.

GUIMARÃES, D. P. Desenvolvimento de um modelo de distribuição diamétrica de passo invariante para prognose e projeção da estrutura de povoamentos de eucalipto. 1994. $160 \mathrm{f}$. Tese (Doutorado em Ciência Florestal) - Universidade Federal de Viçosa, Viçosa, MG, 1994.

HAFLEY, W. L.; SCHREUDER, H. T., Statistical distributions for fitting diameter and height data in even-aged stands. Canadian Journal of Forest Research, v.7, n.1, p.184-487, 1977.

HAYKIN, S. Redes neurais: princípios e prática. Porto Alegre: Bookman, 2001. 900p.

HYINK, D. M. Diameter distribution approaches to growth and yield modeling. In: BROWN, K. M., CLARKE, F.R. (Ed.) Forecasting forest stand dynamics. Ontario: School of Forestry. Lakehead University, 1980. p.138-163.
HYINK, D. M.; MOSER, J. W. A generalized framework for projecting forest yield and stand structure using diameter distributions. Forest Science, v.29, n.1, p.85-95, 1983.

JAIN, A. K.; MAO, J.; MOHIUDDIN, K. M. Artificial neural networks: A Tutorial. Computer, v.29, n.3, p.31-44, 1996.

LEITE, H. G. et al. Avaliação do ajuste das funções Weibull e hiperbólica a dados de povoamentos de eucalipto submetidos a desbaste. Revista Árvore, v.34, n.2, p.305-311, 2010.

LOETSCH, F.; ZOHRER, F.; HALLER, K. Forest inventory. Munchen: BLV Verlagsgesellschaft, 1973. $479 \mathrm{p}$.

NEWBY, M. The properties of moment estimators for the Weibull distribution based on the sample coefûcient of variation. Technometrics, v.22, n.1, p.187-194, 1980.

NOGUEIRA, G. S. et al. Modelo de distribuição diamétrica para povoamentos de Eucalyptus sp. submetidos a desbaste. Revista Árvore, v.29, n.4, p.579-589, 2005.

SIIPILEHTO, J. Improving the accuracy of predicted basal-area diameter distribution in advanced stands by determining stem number. Silva Fennica, v.33, n.4, p.281-301, 1999.

SILVA, M. L. M. Estimação do diâmetro sem casca e diâmetro do cerne para árvores de Tectona grandis linn. utilizando redes neurais artificiais. Monografia. Universidade Federal de Viçosa, Viçosa, MG, 2008.

SOKAL, R. R.; ROHLF, F. J. Biometry. San Francisco: Freeman, 1981. 859 p.

STATSOFT, INC. STATISTICA (data analysis software system), version 10. 2010.

$<$ http:\|www.statsoft.com.br>. 